\title{
FENÔMENOS DE ORDEM-DESORDEM LOCAL EM TURMALINAS NATURAIS E TRATADAS DA SÉRIE SCHORLITA-ELBAITTA
}

\author{
CRISTIANE CASTAÑEDA ${ }^{1,3}$, SIGRID GRIET EECKHOUT $^{2}$, EDDY DE GRAVE $^{2}$, \\ NILSON FRANCISQUINI BOTELHO ${ }^{1} \&$ ANTÔNIO CARLOS PEDROSA SOARES ${ }^{3}$
}

\begin{abstract}
LOCAL ORDER-DISORDER PHENOMENA IN NATURAL AND TREATED TURMALINE OS THE SCHORLITEELBAITE SERIES We report a comparative study between natural and treated green, blue and black color tourmalines from granitic pegmatites of the Minas Gerais state, Brazil, using Electron Microprobe, Electron Paramagnetic Resonance (EPR) and Mössbauer spectroscopy. Electron Microprobe analysis confirms that all studied samples are schorl species. The Electron Paramagnetic Resonance spectra of green and blue tourmaline are characterized by line widths of about 1000 Gauss. These unusual broad lines are probably due to spin-spin interactions caused by the high Fe concentrations and structural disorder. Mössbauer spectra collected at room temperature confirm that iron is dominantly present as $\mathrm{Fe}^{2+}$. It is incorporated at two or three different $\mathrm{Y}$ sites for the green and blue tourmalines, respectively. From those features, we conclude that blue tourmalines are characterized by a high degree of disorder, whereas a smaller degree of disorder is observed in green tourmalines. In addition, heat treatments in oxidizing and reducing atmospheres yielded structural readjustments at the Fe sites, de-hydroxylation, oxidation and generation of a second, different phase.
\end{abstract}

Keywords: tourmaline, Mössbauer spectra, order-disorder.

\begin{abstract}
Resumo Análises de Fe-turmalinas provenientes de pegmatitos graníticos foram feitas usando microssonda eletrônica, ressonância paramagnética eletrônica e espectroscopia Mössbauer. A caracterização química indica que todas as amostras são espécies próximas da schorlita. Os espectros de ressonância paramagnética eletrônica indicam alta concentração de ferro com linhas largas de aproximadamente 1000 Gauss, diagnosticando interações spin-spin e desordem estrutural. Espectros Mössbauer à temperatura ambiente de schorlitas verdes naturais apresentam contribuição de dois $\mathrm{Fe}^{2+} \mathrm{em}$ sítios $\mathrm{Y}$ não equivalentes enquanto nos espectros das azuis, três sítios Y podem ser observados. Esses aspectos registram um maior grau de desordem nas amostras de cor azul e um menor grau, nas verdes. Durante o aquecimento em ambientes oxidante e redutor, observa-se reajuste estrutural dos sítios do $\mathrm{Fe}^{2+}$, processos de desidroxilação e oxidação e a geração de uma fase mineral adicional.
\end{abstract}

Palavras-chave: turmalina, espectro Mössabuer, ordem-desordem.

INTRODUÇÃO As turmalinas são consideradas como lata de lixo da natureza, em função da quantidade de elementos químicos que podem ser incorporados na sua estrutura. Sua grande variação química, resultante da facilidade com que ela agrega os mais variados elementos, é uma ferramenta importante que permite a sua utilização como indicador petrogenético. Em função disso, formam um grupo mineral observado em vários tipos de rochas em que a maioria das espécies pode apresentar variedades gemológicas (Castañeda et al. 2001).

Os minerais do grupo da turmalina cristalizam-se na classe piramidal trigonal do sistema trigonal, cujo grupo espacial é representado por $\underline{\mathrm{R} 3 \mathrm{~m}}$. As dimensões da cela unitária são calculadas por hipóteses das modificações ordenadas e desordenadas das várias espécies e estudadas com base nos diagramas de Epprech (1953) e Donnay \& Barton (1972). Esses minerais podem ser expressos pela fórmula geral $\mathrm{XY}_{3} \mathrm{Z}_{6}\left(\mathrm{BO}_{3}\right)_{3}\left(\mathrm{~T}_{6} \mathrm{O}_{18}\right) \mathrm{V}_{3} \mathrm{~W}$ (Hawthorne \& Henry 1999). Há dois tipos de sítios octaédricos, normalmente chamados de Y e Z. Esse último é menor e mais distorcido que o Y, sendo usualmente ocupado por $\mathrm{Al}^{3+}$. Porém, quantidades significativas de $\mathrm{Fe}^{3+}, \mathrm{Mg}^{2+}, \mathrm{Cr}^{3+} \mathrm{eV}^{3+}$ podem substituir o alumínio (Barton
1969, Foit \& Rosenberg 1979, Burns 1982). O sítio Y permite diversas e extensivas substituições, envolvendo $\mathrm{Fe}^{2+}, \mathrm{Mg}^{2+}, \mathrm{Al}^{3+}, \mathrm{Li}^{+}$, $\mathrm{Fe}^{3+}, \mathrm{Mn}^{2+}, \mathrm{V}^{3+}, \mathrm{Cr}^{3+}$ e $\mathrm{Ti}^{4+}$ (Fortier \& Donnay 1975, Foit \& Rosenberg 1979). O sítio X é ocupado por cátions alcalinos, geralmente $\mathrm{Na}^{+}$, mas também acomoda os íons $\mathrm{Ca}^{2+}, \mathrm{K}^{+}, \mathrm{Mg}^{2+}$ ou ainda pode manter-se vazio (Foit $\&$ Rosenberg 1977). O boro está em coordenação triangular regular e, aparentemente, não sofre substituição (Povondra 1981, MacDonald \& Hawthorne 1995). No sítio tetraédrico (T), o conteúdo de Si pode ser menor que seis átomos por fórmula unitária (apfu), acomodando ainda quantidades suficientes de ${ }^{[4]} \mathrm{Al}$ (MacDonald \& Hawthorne 1995). Finalmente, existem dois sítios para a hidroxila, $\mathrm{V}^{\circ} \mathrm{O}_{3} \mathrm{e} \mathrm{W}^{\circ} \mathrm{O}_{1}$, que são ocupados por ânions monovalentes $\left(\mathrm{OH}, \mathrm{F}^{-}\right)$e ou divalentes $\left(\mathrm{O}^{2-}\right)$. $\mathrm{O}$ sítio $\mathrm{V}$ é geralmente dominado por $\mathrm{OH}$ e $\mathrm{O}^{2-}$, enquanto que o $\mathrm{W}$ pode conter OH, F- e $\mathrm{O}^{2-}$ (Hawthorne \& Henry 1999) ou ainda manter-se vazio (Foit \& Rosenberg 1977).

Fenômenos de ordem-desordem na estrutura da turmalina têm sido objeto de estudo de vários trabalhos (Grice \& Ercit 1993, Hawthorne et al. 1993, Hawthorne 1996, Hawthorne \& Henry 1999, Pieczka 1999, 2000, Bloodaxe et al. 1999). Entretanto, esses fenô-

1 - Instituto de Geociências, Universidade de Brasília, 70910-900, Brasília, Distrito Federal, Brasil.

2 - Department of Subatomic and Radiation Physics, Gent University, Proeftuinstraat 86, B-9000 Gent, Bélgica

3 - Instituto de Geociências, Universidade Federal de Minas Gerais, 30430-120, Belo Horizonte, Minas Gerais, Brasil. e-mail: ccasteneda@igc.ufmg.br 
menos não têm sido aplicados à série elbaíta-schorlita.

Vários mecanismos de substituição podem ocorrer na estrutura da turmalina e são responsáveis pela desordem estrutural. A presença de $\mathrm{O}^{2-}$ no sítio $\mathrm{W}$ controla a ocupação do sítio $\mathrm{Y}$ devido aos requisitos da valência local e comprimento de ligação. Na oxidravita, o arranjo catiônico em torno do $\mathrm{O} 1$ é $3 \mathrm{Y}=2 \mathrm{Al}^{3+}+1 \mathrm{Mg}^{2+}$ para que as regras do somatório de valência sejam satisfeitas, causando uma configuração mais desordenada, uma vez que o Mg está deslocado para o sítio Z e o Al, para o Y (Hawthorne 1996, Hawthorne \& Henry 1999). As substituições catiônicas nos sítios adjacentes $Y$ e Z influenciam suas dimensões e causam um efeito geométrico na estrutura do cristal, o qual pode ser estimado pelos parâmetros estruturais da cela unitária. O desvio dos valores fornece o grau de ordem-desordem dos íons entre os sítios Y e Z. Se uma espécie apresenta diferenças nos parâmetros estruturais em relação ao membro final, ela pode ser considerada desordenada (Pieczka 1999, 2000). Por outro lado, determinadas substituições favorecem uma distribuição ordenada dos íons nos sítios Y e Z. Um exemplo é a presença de $\mathrm{Fe}^{2+}$ no sítio Y, na série schorlita-dravita, impedindo a presença de $\mathrm{Mg}$ no sítio $\mathrm{Z}$, por causa do desvio antitético do O6 requerido na dupla substituição ( $\mathrm{Fe}^{2+}$ no $\mathrm{Y}$ e $\mathrm{Mg}$ no $\mathrm{Z}$ ) (Bloodaxe et al. 1999).

Considerando as hipóteses mencionadas, é de se esperar que a presença de Fe em sítios Y não equivalentes possa servir como uma ferramenta para caracterizar o grau de ordenamento da estrutura, uma vez que um alto grau de ordenamento dos cátions implica em baixo número de sítios Y não equivalentes (Castañeda 2002, Oliveira et al. 2002). Partindo-se dessa premissa, será avaliado o tipo de sítio e o estado de valência do íon Fe por intermédio da espectroscopia Mössbauer em turmalinas do tipo schorlita. O comportamento espectral dessas turmalinas é discutido em termos de ambiente local com o objetivo de caracterizar os fenômenos de ordem e desordem.

MÉTODOS UTILIZADOS Análises pontuais nas amostras foram realizadas em microssonda eletrônica, no laboratório do Instituto de Geociências da Universidade de Brasília utilizando-se equipamento CAMEBAX SX-50 (1990) com 4 espectrômetros WDS e 1 EDS. As condições de operação para todos os elementos foram de $15 \mathrm{KV}$ e $20 \mathrm{nA}$. Os cristais foram analisados com os seguintes padrões: jadeíta $(\mathrm{Na})$, ortoclásio $(\mathrm{K})$, hortonolita ( $\mathrm{Si}, \mathrm{Fe}$, $\mathrm{Mg}, \mathrm{Mn})$, fluorita $(\mathrm{F}), \mathrm{TiO}_{2}(\mathrm{Ti})$, anortita $(\mathrm{Ca}, \mathrm{Al})$, cromita $(\mathrm{Cr}), \mathrm{NiO}$ (Ni), $\mathrm{ZnO}(\mathrm{Zn}), \mathrm{V}_{2} \mathrm{O}_{3}(\mathrm{~V})$ e $\mathrm{Cu}_{2} \mathrm{O}(\mathrm{Cu})$. Os dados químicos para cada cristal correspondem à analise de aproximadamente 20 pontos escolhidos uniformemente em cada cristal. As fórmulas estruturais dos minerais do grupo da turmalina foram calculadas com base em 24,5 ânions, assumindo quantidades estequiométricas de $\mathrm{H}_{2} \mathrm{O}$ e $\mathrm{B}_{2} \mathrm{O}_{3}$, calculados a partir de $\mathrm{OH}^{-}=(4-\mathrm{F})$ átomos por fórmula unitária (apfu), e $\mathrm{B}^{3+}=3$ apfu, respectivamente. A quantidade de $\mathrm{Li}^{+}$atribuída ao sítio cristalográfico $\mathrm{Y}$ foi feita como sendo igual ao somatório ideal do sítio $\mathrm{Y}(=3)$ menos a quantidade dos outros cátions que ocupam o mesmo sítio, ou seja $\mathrm{Li}=3-\mathrm{Y}$ (Burns et al. 1994). O Mn foi considerado como $\mathrm{Mn}^{2+}$. As atribuições dos sítios foram feitas, assumindo que: 1) a deficiência de Si no sítio T é compensada por ${ }^{[4]} \mathrm{Al}$; 2) o sítio $\mathrm{Z}$ é ocupado por ${ }^{[6]} \mathrm{Al}$ e 3 ) os cátions restantes, com exceção de $\mathrm{Na}, \mathrm{Ca}$ e $\mathrm{K}$ no sítio X, ocupam o sítio Y. A distribuição do ferro foi feita por meio da espectrometria Mössbauer.

Os espectros Mössbauer em temperatura ambiente foram coletados em 1024 canais com um espectrômetro convencional de aceleração constante com sinal de referência triangular (CMTE modelo MA350) em rotina no Departamento de Física Subatômica e de radiação da Universidade de Gent, Bélgica. O aumento da velocidade foi de $\sim 0.016 \mathrm{~mm} / \mathrm{s}$ por canal. Uma fonte de $\mathrm{Co}^{57} \mathrm{em}$ rhódio foi usada e a escala da velocidade foi periodicamente calibrada contra uma capa de a-Fe. Todos os valores do desvio isomérico cotado foram relativos ao desvio do a-Fe. O absorvedor consistiu de espécies finamente pulverizadas estendidas uniformemente sobre um suporte e, subseqüentemente, seladas com uma camada orgânica. A espessura foi em média de $\sim 5 \mathrm{mg} / \mathrm{cm}^{2} \mathrm{de}$ Fe natural. A acumulação dos dados foi contínua até que a soma do final da ressonância fosse alcançada. Os espectros foram ajustados com dupletos Lorenzianos simétricos (i.e. espessuras e intensidades iguais de linhas para as duas partes de uma dado dupleto), sem imposição qualquer de restrições adicionais nos parâmetros dos valores a serem ajustados. Os espectros foram também analisados usando o ajuste de distribuição de forma independente (Vandenberghe et al. 1994) usando superposições das distribuições do desdobramento quadrupolar (QSD) de $\mathrm{Fe}^{2+}, \mathrm{Fe}^{3+}$ e $\mathrm{Fe}^{2 \mathrm{n}+}$ (ferro com estado de valência misto), com um único valor de desvio isomérico, diferente, para cada estado de valência. As larguras de linha dos dupletos elementares para cada um dos componentes foram parâmetros reguláveis. A faixa do QSD para o $\mathrm{Fe}^{2+}$ foi de $2,80 \mathrm{~mm} / \mathrm{s}-1,20 \mathrm{~mm} / \mathrm{s}$ (50 valores), para a contribuição do $\mathrm{Fe}^{2 \mathrm{n}+}$ foi $1,20 \mathrm{~mm} / \mathrm{s}-0,50 \mathrm{~mm} / \mathrm{s}$ (16 valores) e para $\mathrm{Fe}^{3+}$ foi de 1,20 $\mathrm{mm} / \mathrm{s}-0,50 \mathrm{~mm} / \mathrm{s}$ (30 valores).

As amostras naturais foram aquecidas em atmosferas oxidante (ar) e redutora $\left(\mathrm{N}_{2}\right)$. O tratamento térmico em ambiente oxidante foi realizado em um forno elétrico de filamento de molibdênio com compartimento isolante, acoplado a uma controladora de temperatura do tipo PID, Eurotherm 818S, com atmosfera não controlada. Foram realizados experimentos com rampeamento de $10 \%$ min até atingir as temperaturas máximas desejadas de $700^{\circ} \mathrm{C}$ e $800^{\circ} \mathrm{C}$, mantidas por 12 horas. $\mathrm{O}$ resfriamento foi controlado da mesma maneira que o aquecimento. $\mathrm{O}$ tratamento térmico em ambiente redutor foi realizado em um forno tubular com resistência $\mathrm{SiC}$ e temperatura controlada com termopar do tipo S (platina/platina $10 \%$ Rd). Trabalhou-se em condições de atmosfera controlada utilizando nitrogênio de alta pureza. Foram utilizados os mesmos processos do tratamento térmico em ambiente oxidante.

RESULTADOS E DISCUSSÕES Composição química Turmalinas verdes, azuis e negras provenientes de pegmatitos graníticos do Distrito Pegmatítico de Araçuaí, Minas Gerais, foram selecionadas para o estudo. A Tabela 1 apresenta a composição química dada pela média de análises de microssonda eletrônica em cada cristal e/ou zona de cor. Os elementos $\mathrm{Si}, \mathrm{Al}, \mathrm{Fe}, \mathrm{Mn}, \mathrm{Mg}, \mathrm{Zn}$, $\mathrm{Ti}, \mathrm{Li}^{*}, \mathrm{Na}, \mathrm{Ca}, \mathrm{K}$, e F foram considerados maiores ou subordinados (*o Li não foi analisado por microssonda eletrônica). Com poucas exceções, os cristais de turmalina contêm baixos teores de $\mathrm{Mg}, \mathrm{Zn}$ e Ti. $\mathrm{SiO}_{2}$ e $\mathrm{Al}_{2} \mathrm{O}_{3}$ estão coerentes com a composição química ideal desses minerais, assim como cálcio e os álcalis.

A avaliação dos dados químicos dos elementos maiores caracteriza as espécies como sendo schorlita que, devido a variações nos conteúdos de $\mathrm{Fe}_{\text {total }}$, podem ser divididas em schorlita pura e membros intermediários. Os cristais de schorlita são predominantemente pretos e azul-marinho, de base larga (megacristais de ate $15 \mathrm{~cm}$ ) e prisma trigonal curto, representados pelas amostras MR4, Pi2 e SC2a. Exibem uma variação dos conteúdos de FeO(t) entre 8 e $14 \%$, livres de concentrações significativas de outros elementos cromóforos. Os membros intermediários têm cores desde azul, passando para matizes esverdeados até o verde-garrafa, e são repre- 
Tabela 1 - Análises químicas das amostras de turmalinas estudadas e suas fórmulas estruturais. $\mathrm{Li}_{2} \mathrm{O}_{\text {e }} \mathrm{H}_{2} \mathrm{O}$ foram calculados de acordo com explicação no texto.

\begin{tabular}{|c|c|c|c|c|c|c|c|c|c|c|c|c|}
\hline & \multicolumn{2}{|c|}{ Preta } & \multirow{2}{*}{$\begin{array}{c}\text { Azul } \\
\text { marinbo } \\
\text { SC2a }\end{array}$} & \multicolumn{2}{|c|}{ Azul } & \multirow{2}{*}{$\begin{array}{l}\text { Azul } \\
\text { claro } \\
\text { CAAC }\end{array}$} & \multicolumn{3}{|c|}{ Verde escuro } & \multirow{2}{*}{$\begin{array}{l}\text { Verde } \\
\text { grama } \\
\text { CAVG }\end{array}$} & \multicolumn{2}{|c|}{$\begin{array}{l}\text { Verde } \\
\text { claro }\end{array}$} \\
\hline & MR4 & $\mathrm{Pi} 2$ & & CA3 & PD & & & A1VG & & & CAVC & CAEV \\
\hline $\mathrm{SiO}_{2}$ & 35,39 & 36,03 & 36,37 & 36,83 & 37,07 & 37,38 & 35,83 & 37,24 & 37,07 & 36,94 & 37,32 & 37,43 \\
\hline $\mathrm{TiO}_{2}$ & 0,28 & $\mathrm{O}, \mathrm{II}$ & 0,02 & O,OI & 0,02 & O,OI & $\mathrm{O}, \mathrm{I} 3$ & O,IO & 0,09 & 0,08 & O,OI & 0,02 \\
\hline $\mathrm{Al}_{2} \mathrm{O}_{3}$ & 34,97 & 37,35 & 36,90 & 38,43 & 37,67 & 38,74 & 36,50 & 38,44 & 38,47 & 38,57 & 39,53 & $39,7 \mathrm{I}$ \\
\hline $\mathrm{FeO}$ & $\mathrm{I} 2, \mathrm{O} 3$ & 8,36 & 7,83 & 4,59 & 6,98 & 2,64 & 8,46 & 3,89 & 2,98 & 3,23 & 1,26 & $\mathrm{I}, 42$ \\
\hline $\mathrm{Fe}_{2} \mathrm{O}_{3}$ & $\mathrm{I}, 4 \mathrm{O}$ & $0,8 \mathrm{I}$ & 0,65 & 0,00 & 0,00 & 0,00 & 0,00 & 0,00 & 0,00 & 0,00 & 0,00 & 0,00 \\
\hline $\mathrm{MgO}$ & $\mathrm{I}, \mathrm{IO}$ & 0,26 & 0,04 & O,OI & 0,04 & O,OI & 0,02 & $\mathrm{O}, \mathrm{II}$ & 0,54 & 0,45 & 0,00 & 0,00 \\
\hline $\mathrm{MnO}$ & 0,26 & 0,20 & 0,38 & 2,39 & 0,53 & $2, \mathrm{OI}$ & $\mathrm{I}, \mathrm{O} 4$ & $\mathrm{I}, 62$ & $\mathrm{I}, 45$ & $\mathrm{I}, 32$ & 2,32 & 2,30 \\
\hline $\mathrm{ZnO}$ & O,I9 & $0,4 \mathrm{I}$ & 0,44 & 0,05 & O,I4 & 0,20 & 0,58 & 0,24 & 0,08 & 0,06 & $\mathrm{O}, \mathrm{I} 2$ & O,I4 \\
\hline $\mathrm{CaO}$ & 0,09 & 0,07 & $\mathrm{O}, \mathrm{II}$ & 0,28 & $\mathrm{O}, \mathrm{I} 2$ & 0,33 & O,I6 & 0,90 & 0,40 & 0,55 & 0,49 & 0,53 \\
\hline $\mathrm{Na}_{2} \mathrm{O}$ & $\mathrm{I}, 6 \mathrm{O}$ & 2,16 & 3,12 & 2,58 & 2,75 & $2,5 I$ & 2,95 & 2,78 & 3,08 & 2,45 & 2,32 & 2,34 \\
\hline $\mathrm{K}_{2} \mathrm{O}$ & 0,05 & 0,03 & 0,03 & 0,03 & 0,04 & 0,02 & 0,04 & 0,02 & 0,05 & 0,03 & O,OI & 0,02 \\
\hline $\mathrm{Li}_{2} \mathrm{O}$ & 0,29 & 0,87 & $\mathrm{I}, 23$ & I,O6 & I,I6 & $\mathrm{I}, 45$ & $\mathrm{I}, \mathrm{O} 6$ & I,5O & I,49 & $\mathrm{I}, 35$ & $\mathrm{I}, 44$ & $\mathrm{I}, 44$ \\
\hline $\mathrm{H}_{2} \mathrm{O}+$ & 3,57 & 3,38 & $3,3 I$ & 3,68 & 3,69 & 3,70 & 3,34 & 3,33 & 3,44 & 3,69 & 3,69 & 3,72 \\
\hline $\mathbf{F}$ & 0,15 & 0,62 & 0,82 & O,OI & O,OI & nd & 0,67 & 0,87 & 0,60 & nd & O,OI & nd \\
\hline $\mathrm{O}=\mathrm{F}$ & 0,06 & 0,26 & 0,35 & 0,00 & 0,00 & 0,00 & 0,28 & 0,37 & 0,25 & 0,00 & 0,00 & 0,00 \\
\hline TOTAL & 91,35 & 90,41 & 90,90 & 89,95 & 90,22 & 89,00 & 90,51 & 90,67 & 89,49 & 88,72 & 88,51 & 89,05 \\
\hline Si4+ & 5,83 & 5,87 & 5,89 & 5,99 & 6,02 & 6,05 & 5,85 & 5,97 & 5,96 & 6,00 & 6,05 & 6,03 \\
\hline $\mathrm{Al}^{3+}$ & O,I7 & $\mathrm{O}, \mathrm{I} 3$ & O,II & 0,02 & O,OI & 0,00 & $\mathrm{O}, \mathrm{I} 5$ & 0,03 & O,II & O,OI & 0,00 & 0,00 \\
\hline T Si & 6,00 & 6,00 & 6,00 & $6, \mathrm{OI}$ & 6,03 & 6,05 & 6,00 & 6,00 & 6,07 & $6, \mathrm{OI}$ & 6,06 & 6,03 \\
\hline $\mathrm{Al}^{3+}$ & 6,00 & 6,00 & 6,00 & 6,00 & 6,00 & 6,00 & 6,00 & 6,00 & 6,00 & 6,00 & 6,00 & 6,00 \\
\hline TZ & 6,00 & 6,00 & 6,00 & 6,00 & 6,00 & 6,00 & 6,00 & 6,00 & 6,00 & 6,00 & 6,00 & 6,00 \\
\hline $\mathrm{Fe}^{+2}$ & $\mathrm{I}, 66$ & $\mathrm{I}, \mathrm{I} 4$ & I,O6 & 0,63 & 0,95 & 0,36 & I,I6 & 0,49 & 0,40 & 0,44 & O,I7 & O,I9 \\
\hline $\mathrm{Fe}^{3+}$ & O,I7 & O,IO & 0,08 & 0,00 & 0,00 & 0,00 & 0,00 & 0,00 & 0,00 & 0,00 & & 0,00 \\
\hline $\mathrm{Mg}^{2+}$ & 0,27 & 0,06 & O,OI & 0,00 & O,OI & 0,00 & 0,00 & 0,04 & $\mathrm{O}, \mathrm{I} 3$ & O,II & 0,00 & 0,00 \\
\hline $\mathrm{Mn}^{2+}$ & 0,04 & 0,03 & 0,05 & 0,33 & 0,07 & 0,28 & O,I4 & $0,2 \mathrm{I}$ & 0,20 & O,I8 & 0,32 & $\mathrm{O}, 3 \mathrm{I}$ \\
\hline $\mathrm{Ti}^{4+}$ & 0,03 & O,OI & 0,00 & 0,00 & 0,00 & 0,00 & 0,02 & O,OI & O,OI & O,OI & 0,00 & 0,00 \\
\hline $\mathrm{Zn}^{2+}$ & 0,02 & 0,05 & 0,05 & $\mathrm{O}, \mathrm{OI}$ & 0,02 & 0,02 & 0,07 & 0,02 & O,OI & O,OI & O,OI & 0,02 \\
\hline $\mathrm{Li}^{+}$ & O,I9 & 0,57 & 0,80 & 0,69 & 0,76 & 0,95 & 0,73 & 0,99 & $\mathrm{I}, \mathrm{O} 7$ & 0,88 & 0,94 & 0,93 \\
\hline $\mathrm{Al}^{3+}$ & 0,62 & $\mathrm{I}, \mathrm{O} 4$ & 0,95 & $\mathrm{I}, 34$ & I,I9 & $\mathrm{I}_{3} 39$ & 0,88 & I, 23 & I,I8 & $\mathrm{I}_{3} 37$ & 1,56 & I,54 \\
\hline TY & 3,00 & 3,00 & 3,00 & 3,00 & 3,00 & 3,00 & 3,00 & 3,00 & 3,00 & 3,00 & 3,00 & 3,00 \\
\hline $\mathrm{Ca}^{2+}$ & 0,02 & $\mathrm{O}, \mathrm{OI}$ & 0,02 & 0,05 & 0,02 & 0,06 & 0,03 & O,I7 & 0,07 & O,IO & 0,09 & 0,09 \\
\hline $\mathrm{Na}^{+}$ & $0,5 \mathrm{I}$ & 0,68 & 0,98 & $0,8 \mathrm{I}$ & 0,87 & 0,79 & 0,94 & 0,85 & 0,96 & 0,77 & 0,73 & 0,73 \\
\hline $\mathrm{K}^{+}$ & O,OI & O,OI & O,OI & O,OI & O,OI & 0,00 & O,OI & 0,00 & O,OI & O,OI & 0,00 & 0,00 \\
\hline $\mathbf{T} \mathbf{X}$ & 0,54 & 0,70 & $\mathrm{I}, \mathrm{OO}$ & 0,87 & 0,90 & 0,85 & 0,97 & $\mathrm{I}, \mathrm{O} 2$ & $\mathrm{I}, \mathrm{O} 4$ & 0,87 & 0,82 & 0,83 \\
\hline $\mathrm{OH}-$ & 3,92 & 3,68 & 3,58 & 3,99 & 4,00 & 4,00 & 3,65 & 3,56 & 3,69 & 4,00 & 4,00 & 4,00 \\
\hline F- & 0,08 & 0,32 & 0,42 & O,OI & 0,00 & 0,00 & 0,35 & 0,44 & $0,3 \mathrm{I}$ & 0,00 & 0,00 & 0,00 \\
\hline $\mathrm{T} \mathrm{OH}$ & 4,00 & 4,00 & 4,00 & 4,00 & 4,00 & 4,00 & 4,00 & 4,00 & 4,00 & 4,00 & 4,00 & 4,00 \\
\hline
\end{tabular}

sentados pelas amostras CA3, PD, CAAC, CAEV, CAVC, CA1VG, CAVG, as quais podem apresentar qualidade gemológica. São amostras de cristais com dimensões variáveis entre 3 a $6 \mathrm{~cm}$ de comprimento e 0,5 a $4 \mathrm{~cm}$ de largura. Trata-se de membros intermediários, ora mais próximos da molécula da elbaíta, ora da schorlita, com ferro e manganês na fórmula estrutural. Os teores de ferro variam desde 1,25\% em amostras claras até próximos a 7,0\% em escuras.

Caracterização do ferro nas amostras naturais A principal técnica escolhida para o estudo dessas espécies, a espectroscopia Mössbauer (MS), permite não só o exame do estado de valência do ferro, como também a distinção da ocupação dos diferentes sítios. A distinção do número de sítios não equivalentes do ferro permite a caracterização do grau de ordem e desordem da estrutura, o que pode favorecer diferentes mecanismos de causas de cor. Além disso, a razão $\mathrm{Fe}^{3+} / \mathrm{Fe}^{2+}$ é um bom indicador das condições de fugacidade do oxigênio que prevaleceram durante a cristalização da turmalina (Oliveira et al. 2002). Diferentes condições de fugacidade podem favorecer ou permitir diferentes estados de valência dos metais de transição.

Em geral, os espectros Mössbauer de turmalinas são difíceis de ajustar devido à possibilidade da presença de vários dupletos de $\mathrm{Fe}^{2+}$, e/ou $\mathrm{Fe}^{2 \mathrm{n}+}$ (valência mista) e/ou $\mathrm{Fe}^{3+}$, os quais podem estar fortemente superpostos. Dyar et al. (1998) reavaliaram os dados dos parâmetros hiperfinos de turmalinas registrados na literatura e apontaram um enorme espalhamento nos valores, em parte resultante das diferentes maneiras de usar o ajuste do espectro. Infeliz- 
mente, nenhum consenso sobre modelos apropriados para serem aplicados foi encontrado. No primeiro estágio deste trabalho, todos os espectros foram ajustados com superposições de um número de dupletos Lorentzianos simétricos, sem imposição para qualquer restrição nos valores dos parâmetros a serem ajustados. Essa primeira tentativa de interpretar os espectros foi feita para revelar um comportamento global, satisfatório, dos parâmetros Mössbauer das amostras examinadas. Os resultados desse procedimento de ajuste para algumas amostras selecionadas estão nas figuras 1 e 2 e os resultados numéricos constam da Tabela 2. Como pode ser visto, as formas das linhas calculadas reproduzem adequadamente as experimentais. Além disso, os parâmetros Mössbauer estão coerentes com aqueles registrados na literatura (Dyar et al. 1998).

Embora o procedimento de ajuste pareça aceitável inicialmente, as linhas extremamente largas de alguns dupletos de $\mathrm{Fe}^{2+}$ e $\mathrm{Fe}^{3+}$ $(\sim 0.4 \mathrm{~mm} / \mathrm{s})$ sugerem a presença de componentes de ferro adicionais. A existência de diferentes configurações vizinhas no ambiente local do ferro em turmalinas naturais implica em uma distribuição, mais ou menos extensa, dos valores do desdobramento quadrupolar, a qual pode ser esperada para vários sítios do ferro não equivalentes.

Rancourt (1994) discutiu, previamente, a existência de tais efeitos na distribuição dos valores do desdobramento quadrupolar em vários minerais. Como conseqüência, em um estágio subseqüente do presente trabalho, todos os espectros Mössbauer foram descritos com o uso do modelo QSD (distribuição dos valores dos desdobramento quadrupolar). Comparações entre ambos modelos, usando o critério convencional para "bons ajustes" ba- seado no $c^{2}$ (Ruby 1973), mostraram que nenhuma melhora foi obtida com o método da distribuição. Para algumas amostras, o modelo QSD falhou, visto que nenhum ajuste convergente pode ser estabelecido. Além disso, a diferença entre a probabilidade máxima dos valores dos desdobramentos quadrupolares do $\mathrm{Fe}^{2+}$ do sítio Y1 e Y2 (vide atribuições), cujo significado é mais relevante do que a média do desdobramento quadrupolar, é menor que 0,2 $\mathrm{mm} / \mathrm{s}$. Com o objetivo de testar o modelo Lorentziano, os parâmetros hiperfinos obtidos do ajuste QSD foram usados como valores iniciais. Cálculos subseqüentes do MS resultaram em parâmetros hiperfinos comparáveis e valores de $c^{2}$ semelhantes aos obtidos inicialmente do modelo Lorentziano.

Duas diferentes interpretações para os dupletos de ferro em turmalinas têm sido sugeridas na literatura. A maioria dos autores (Burns 1972, Da Costa et al. 1998, Fuchs et al. 1998) atribui o dupleto com maior valor do desdobramento quadrupolar $\mathrm{D} E_{\mathrm{O}}$ $(\sim 2,28-2,47 \mathrm{~mm} / \mathrm{s})$ ao $\mathrm{Fe}^{2+}$ no sítio $\mathrm{Y}$, e o menor $\mathrm{D} E_{\mathrm{Q}}(\sim 1,60 \mathrm{~mm} / \mathrm{s})$, ao $\mathrm{Fe}^{2+}$ no sítio $\mathrm{Z}$. Uma interpretação alternativa em relação aos subespectros do ferro é baseada na hipótese de que todo o $\mathrm{Fe}$ deve estar localizado no sítio Y, uma vez que a presença de Fe no Zé improvável cristalograficamente (Pieczka et al. 1997, Dyar et al. 1998). Além disso, resultados mais recentes de refinamento estrutural por difratometria de raios $\mathrm{X}$ têm suportado a proposta de que o $\mathrm{Al}$ e $\mathrm{Mg}$ ocupam exclusivamente o sítio $\mathrm{Z}$, enquanto todo $\mathrm{Fe}$ entra no sítio Y (Bloodaxe et al. 1999, Hawthorne et al. 1993). Por essas razões, a segunda interpretação será considerada como a mais apropriada.

Estudos de espectroscopia óptica (Faye et al. 1974, Mattson \& Rossman 1987a,b) e Mössbauer (Ferrow et al. 1988, Da Costa et

Tabela 2 - Parâmetros Hiperfinos derivados dos espectros Mössbauer das Fe-turmalinas estudadas. Desvio isomérico, d (mm/s, relativo ao a-Fe), desdobramento quadrupolar, $D E_{Q}(\mathrm{~mm} / \mathrm{s})$, largura a meia altura, $G(\mathrm{~mm} / \mathrm{s})$ e área relativa, $R A(\%)$.

\begin{tabular}{|c|c|c|c|c|c|c|}
\hline COR & Amostras & $\delta$ & $\Delta E_{\mathrm{Q}}$ & $\Gamma$ & $\mathbf{R A}$ & A T R IB U IÇã O \\
\hline \multirow{9}{*}{$\begin{array}{l}\frac{\pi}{5} \\
\ddot{z} \\
\ddot{z}\end{array}$} & MR4* & $1.12(4)$ & $2.50(6)$ & $0.26(1)$ & $37(2)$ & $\mathrm{Fe}^{2+} \mathrm{Y} 1$ \\
\hline & & $1.11(4)$ & $2.21(6)$ & $0.30(1)$ & $33(2)$ & $\mathrm{Fe}^{2+} \mathrm{Y} 2$ \\
\hline & & $1.11(4)$ & $1.63(6)$ & $0.49(1)$ & $13(2)$ & $\mathrm{Fe}^{2+} \mathrm{Y} 3$ \\
\hline & & $0.41(4)$ & $0.50(6)$ & $0.34(1)$ & $2(2)$ & $\mathrm{Fe}^{3+} \mathrm{Y}$ \\
\hline & & $0.86(4)$ & $1.29(6)$ & $0.49(1)$ & $15(2)$ & $\mathrm{Fe}^{2+}-\mathrm{Fe}^{3+}$ \\
\hline & Pi2n* & $1.11(2)$ & $2.49(4)$ & $0.29(1)$ & $33(2)$ & $\mathrm{Fe}^{2+} \mathrm{Y} 1$ \\
\hline & & $1.13(2)$ & $2.26(4)$ & $0.48(1)$ & $56(2)$ & $\mathrm{Fe}^{2+} \mathrm{Y} 2$ \\
\hline & & $0.75(2)$ & $2.04(4)$ & $0.71(1)$ & $6(2)$ & $\mathrm{Fe}^{2+}-\mathrm{Fe}^{3+}$ \\
\hline & & $0.47(2)$ & $0.24(4)$ & $0.34(1)$ & $5(2)$ & $\mathrm{Fe}^{3+} \mathrm{Y}$ \\
\hline \multirow{4}{*}{ 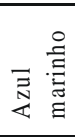 } & SC $2 a^{*}$ & $1.10(4)$ & $2.38(6)$ & $0.27(1)$ & $78(2)$ & $\mathrm{Fe}^{2+} \mathrm{Y} 1$ \\
\hline & & $1.22(4)$ & $2.32(6)$ & $0.34(1)$ & $12(2)$ & $\mathrm{Fe}^{2+} \mathrm{Y} 2$ \\
\hline & & $0.81(4)$ & $1.76(6)$ & $0.66(1)$ & $6(2)$ & $\mathrm{Fe}^{2+}-\mathrm{Fe}^{3+}$ \\
\hline & & $0.47(4)$ & $0.36(6)$ & $0.34(1)$ & $4(2)$ & $\mathrm{Fe}^{3+} \mathrm{Y}$ \\
\hline \multirow{9}{*}{ 崩 } & PD & $0,961(2)$ & $2,416(4)$ & $0,38(1)$ & $66(2)$ & $\mathrm{Fe}^{2+} \mathrm{Y} 1$ \\
\hline & & $1,029(2)$ & $2,279(4)$ & $0,38(1)$ & $28(2)$ & $\mathrm{Fe}^{2+} \mathrm{Y} 2$ \\
\hline & & $0,97(2)$ & $1,50(4)$ & $0,43(1)$ & $6(2)$ & $\mathrm{Fe}^{2+} \mathrm{Y} 3$ \\
\hline & CA3 & $0,974(4)$ & $2,396(4)$ & $0,38(1)$ & $86(2)$ & $\mathrm{Fe}^{2+} \mathrm{Y} 1$ \\
\hline & & $1,117(4)$ & $2,144(4)$ & $0,26(1)$ & $8(2)$ & $\mathrm{Fe}^{2+} \mathrm{Y} 2$ \\
\hline & & $1,010(4)$ & $1,482(4)$ & $0,37(1)$ & $6(2)$ & $\mathrm{Fe}^{2+} \mathrm{Y} 3$ \\
\hline & $\mathrm{CAAC}$ & $1,075(2)$ & $2,395(4)$ & $0,36(1)$ & $86(2)$ & $\mathrm{Fe}^{2+} \mathrm{Y} 1$ \\
\hline & & $1,218(2)$ & $2,119(4)$ & $0,27(1)$ & $9(2)$ & $\mathrm{Fe}^{2+} \mathrm{Y} 2$ \\
\hline & & $1,116(2)$ & $1,503(4)$ & $0,28(1)$ & $5(2)$ & $\mathrm{Fe}^{2+} \mathrm{Y} 3$ \\
\hline \multirow{6}{*}{$\begin{array}{l}\stackrel{0}{0} \\
> \\
>\end{array}$} & $\mathrm{L} 2 \mathrm{v}^{*}$ & $1.11(2)$ & $2.39(4)$ & $0.37(1)$ & $83(2)$ & $\mathrm{Fe}^{2+} \mathrm{Y} 1$ \\
\hline & & $1.21(2)$ & $2.14(4)$ & $0.42(1)$ & $17(2)$ & $\mathrm{Fe}^{2+} \mathrm{Y} 2$ \\
\hline & CAVC & $1,077(2)$ & $2,403(4)$ & $0,34(1)$ & $73(2)$ & $\mathrm{Fe}^{2+} \mathrm{Y} 1$ \\
\hline & & $1,181(2)$ & $2,071(4)$ & $0,59(1)$ & $27(2)$ & $\mathrm{Fe}^{2+} \mathrm{Y} 2$ \\
\hline & CAV G & $0,979(2)$ & $2,401(4)$ & $0,34(1)$ & $86(2)$ & $\mathrm{Fe}^{2+} \mathrm{Y} 1$ \\
\hline & & $1,033(2)$ & $1,739(4)$ & $0,43(1)$ & $14(2)$ & $\mathrm{Fe}^{2+} \mathrm{Y} 2$ \\
\hline
\end{tabular}

*Parâmetros Hiperfinos são de Castañeda (1997), atribuições dos sítios, deste estudo. 
al. 1998) têm mostrado que mecanismos de transferência de carga $\mathrm{Fe}^{2+} \ll \mathrm{Fe}^{3+}$ podem ocorrer na turmalina. $\mathrm{Na}$ espectroscopia Mössbauer, o subespectro com valores intermediários do desvio isomérico, $\mathrm{d}$, e do desdobramento quadrupolar, $\mathrm{D} E_{\mathrm{O}},(\mathrm{d}=0,86 \mathrm{~mm} /$ $\mathrm{s}$ e $\mathrm{D} E_{\mathrm{Q}}=1,29 \mathrm{~mm} / \mathrm{s}$ ) está associado com átomos de ferro envolvidos em tais processos de troca eletrônica (Ferrow et al. 1988).

Os picos de $\mathrm{Fe}^{3+}$ nas turmalinas estão sobrepostos por picos dos dupletos de $\mathrm{Fe}^{2+} \mathrm{em}$ mais baixa velocidade. Consequentemente, os dados isolados de Mössbauer não permitem fazer uma distinção entre os sítios octaédricos ${ }^{\left[{ }^{[}\right]} \mathrm{Fe}^{3+} \mathrm{e}^{[\mathrm{Z}]} \mathrm{Fe}^{3+}$, ficando a partição dos íons férricos, nos respectivos sítios, impossível de ser avaliada (Dyar et al. 1998). Entretanto, segundo dados de difratometria de raios X (Bloodaxe et al. 1999, Hawthorne et al. 1993), a atribuição de $\mathrm{Fe}^{3+}$ ao sítio Y é mais plausível. Analogamente, a troca de elétrons associados a dupletos com valores intermediários implica em trajetórias de troca de ${ }^{[\mathrm{Y}]} \mathrm{Fe}^{2+}{ }^{2 \mathrm{Y}]} \mathrm{Fe}^{3+}$, na qual a distância $\mathrm{Fe}$ Fe é suficientemente pequena para possibilitar a transferência de carga. Finalmente, dupletos que correspondem a ${ }^{[\mathrm{T}]} \mathrm{Fe}^{3+}$ (sítios

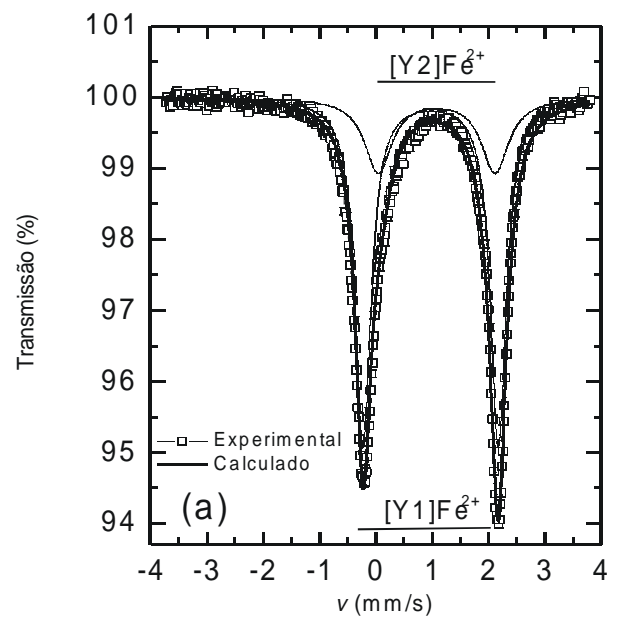

tetraédricos), que têm sido, recentemente, registrados nos espectros Mössbauer de algumas turmalinas (Dyar et al. 1998) não foram registrados nos espectros das amostras estudadas.

Nas turmalinas analisadas, três diferentes tipos de espectro Mössbauer são distinguidos: i) espectros contendo dois componentes de dupletos $\mathrm{Fe}^{2+}$, ii) espectros contendo três componentes de $\mathrm{Fe}^{2+}$ (Fig.1) e iii) espectros contendo três componentes de $\mathrm{Fe}^{2+}$ e pequenas contribuições de componentes de ferro com estado de valência misto e $\mathrm{Fe}^{3+}$. (Fig. 2).

No primeiro tipo, os dois dupletos de ferro podem ser atribuídos $\mathrm{ao}^{[\mathrm{Y}]} \mathrm{Fe}^{2+}\left(\mathrm{D} E_{\mathrm{O} 1}=2,40 \mathrm{~mm} / \mathrm{s} \mathrm{e} \mathrm{DE} E_{\mathrm{O} 2}=2,14 \mathrm{~mm} / \mathrm{s}\right)$ com diferentes coordenações do ambiente local, arbitrariamente designados Y1 e Y2. Nesse aspecto, podem existir outros sítios Y não equivalentes presentes, mas são, presumivelmente, menos abundantes já que sua presença não afeta, significantemente, os dados dos componentes dominantes. As amostras representativas são, exclusivamente, os membros intermediários que apresentam a cor verde (Tab. 2).

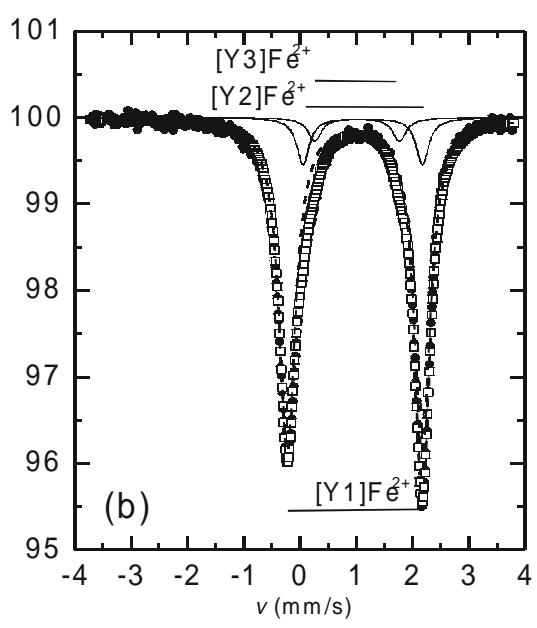

Figura 1 - Espectro Mössbauer sob temperatura ambiente (TA): membro intermediário verde (a), membro intermediário azul (b), ambos naturais.

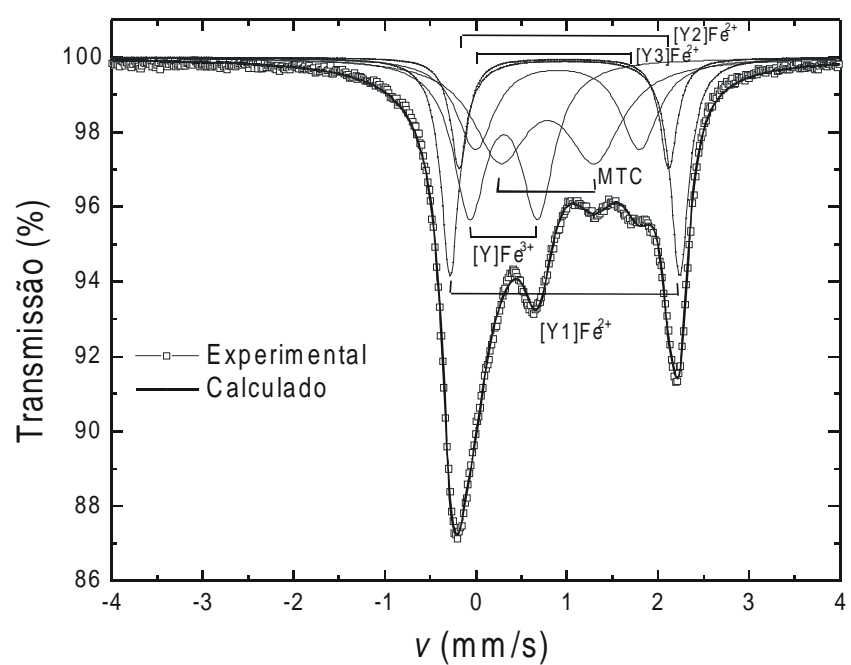

Figura 2 - Espectro Mössbauer sob temperatura ambiente (TA) de uma schorlita natural. (MTC $\mathrm{Fe}^{2+}-\mathrm{Fe}^{3+}$, componentes mistos)
No segundo tipo de espectro, os dois dupletos mais externos podem ser similarmente designados por $\mathrm{Y} 1 \mathrm{e} \mathrm{Y} 2$. O terceiro dupleto de $\mathrm{Fe}^{2+}\left(\mathrm{D} E_{\mathrm{O}}=1,63-1,48 \mathrm{~mm} / \mathrm{s}\right)$ é atribuído ao $\mathrm{Fe}^{2+}$ em um outro sítio Y com diferente ambiente de coordenação local, designado, arbitrariamente $\mathrm{Y} 3$. $\mathrm{O}$ menor valor de $\mathrm{D} E_{\mathrm{O}}$ do $\mathrm{Y} 3$ indica maior distorção do sítio em relação ao Y1 e Y2. Amostras que apresentam esses componentes dominantes são membros intermediários de cor azul (Tab. 2) e algumas schorlitas.

No terceiro tipo, o valor do desdobramento quadrupolar do dupleto férrico $\left(\mathrm{Fe}^{3+}\right)$, presente exclusivamente nas schorlitas mais escuras, indica sua localização em sítio octaédrico distorcido, provavelmente no sítio Y como argumentado anteriormente. Esse íon também foi identificado no espectro de ressonância paramagnética eletrônica (Fig. 3) que revela uma linha característica em g=4,31, além de outras não resolvidas e muito largas, que podem ser atribuídas ao íon $\mathrm{Fe}^{3+}$ localizado em um sítio octaédrico fortemente distorcido. O alargamento das demais linhas de ressonância paramagnética eletrônica ( 1000 Gauss) é, provavelmente, devido às interações spin-spin causadas pelas altas concentrações de ferro e pelo rebaixamento da simetria do sítio, o que parece indicar fenômenos de desordem.

A distribuição de $\mathrm{Fe}^{2+}$ e $\mathrm{Fe}^{3+}$ proposta na fórmula estrutural 
(Tab. 1) foi calculada a partir dos valores ajustados das áreas relativas dos respectivos dupletos, levando em conta a diferença da média estabelecida nas frações Mössbauer para as espécies de $\mathrm{Fe}^{3+}$ e $\mathrm{Fe}^{2+}$ em silicatos (De Grave \& Van Alboom 1991). Os dupletos de ferro com estado de valência misto, tiveram uma metade de sua área relativa atribuída ao $\mathrm{Fe}^{2+}$ e outra metade ao $\mathrm{Fe}^{3+}$. $\mathrm{A}$ correlação de $\sim 5 \%$, em favor dos conteúdos de $\mathrm{Fe}^{2+}$ para as frações do recuo livre não equivalentes, é estimada e considerada razoável.

Juntamente com a presença considerável de ferro no estado misto, a distribuição do $\mathrm{Fe}^{2+}$ em sítios $\mathrm{Y}$ não equivalentes sugere que o mecanismo de transferência de carga é o principal fenômeno ocorrido nas amostras negras e/ou muito escuras. O maior número de sítios Y não equivalentes e a extensa faixa de distribuição dos valores do desdobramento quadrupolar encontrada indicam grau de desordem da estrutura que pode favorecer o condicionamento de determinados centros cromóforos. Esses aspectos estruturais estão diretamente relacionados com a compensação de cargas diferentes, devido à heterogeneidade do ambiente local. De acordo com essa premissa, os diferentes comportamentos dos componentes dominantes dos dupletos de ferro observados nos dois conjuntos de cores, azul e verde, podem ser indícios de diferentes construções de centros cromóforos relativos ao $\mathrm{Fe}^{2+}$.

Caracterização do ferro após aquecimento As amostras, quando submetidas a aquecimento, apresentam espectros Mössbauer distintos. A Tabela 3 apresenta os parâmetros Mössbauer depois do tratamento térmico em ambiente oxidante a $700^{\circ} \mathrm{C}$ e a figura 4 mostra os respectivos espectros.

No espectro obtido após o tratamento térmico em ambiente oxidante, fica evidente a contribuição de $\mathrm{Fe}^{3+}$ na amostra de turmalina azul, concomitante com o desaparecimento do dupleto do ${ }^{\left[{ }^{[} 3\right]} \mathrm{Fe}^{2+} \mathrm{e}$ o decréscimo da área do dupleto do ${ }^{\left[{ }^{1} 1\right]} \mathrm{Fe}^{2+}$, diagnosticando processo de oxidação durante o aquecimento (Figs. 1b e 4a). Além disso, a área relativa do ${ }^{\left[\mathrm{Y}^{2}\right]} \mathrm{Fe}^{2+}$ aumenta significativamente, diagnosticando reajuste estrutural entre os sítios Y do ferro. A estrutura responde à oxidação do $\mathrm{Fe}^{2+}$ por mudanças envolvendo ângulo e distância de ligação, o que explicaria as modificações dos parâmetros hiperfinos do $\mathrm{Fe}^{2+}$ no Y1 e Y2. Segundo Castañeda (2002), a análise termogravimétrica dessa amostra registra uma perda de massa endotérmica na primeira fase de aquecimento que pode ser atribuída à liberação da água adsorvida. Durante esse processo, parte dessa água pode ser quebrada, produzindo $\mathrm{OH}^{-}+\mathrm{H}^{+}$. Um elétron do $\mathrm{Fe}^{2+}$ pode ser capturado pelo $\mathrm{H}^{+}$, formando um centro de captura de elétron $\mathrm{H}^{\circ}$, mantendo o equilíbrio de carga e liberando o $\mathrm{OH}^{-}$produzido. Esse processo pode ser designado por desidrogenação + oxidação (Ishida 1998, Bonazzi $\&$ Bindi 1999) e pode ser descrito esquematicamente da seguinte forma:

$$
\mathrm{Fe}^{2+}+\text { Temperatura }{ }^{\circledR} \mathrm{Fe}^{3+}+\mathrm{e}^{-} \text {(hole center) }
$$

$\mathrm{H}_{2} \mathrm{O}+$ Temperatura ${ }^{\circledR} \mathrm{OH}^{-}+\mathrm{H}^{+}+\mathrm{e}^{-}{ }^{\circledR} \mathrm{H}^{\mathrm{O}}$ (electron center)

$\mathrm{O}$ espectro Mössbauer da amostra verde tratada mostra uma terceira contribuição de $\mathrm{Fe}^{2+}$ em outro sítio Y não equivalente, Y3. Em comparação com a amostra natural, a área do dupleto do ${ }^{[\mathrm{Y} 1]} \mathrm{Fe}^{2+}$ decresce ao passo que a do ${ }^{\left[{ }^{2}\right]} \mathrm{Fe}^{2+}$ aumenta superficialmente (Figs. 1a e 4b). Analogamente à espécie azul, ocorre reajuste estrutural. A análise termogravimétrica da amostra verde revela pequena perda de massa endotérmica em temperatura anterior ao pico da desidroxilação (Castañeda 2002). Nesse aspecto, importantes questões podem ser levantadas: por quê, na turmalina verde, o ferro não é oxidado como na azul? Isto aconteceria sob temperaturas mais elevadas ou os teores de água adsorvida são insignificantes em relação aos da espécie azul, desfavorecendo a oxidação + desidrogenação? Um fato estabelecido é que o teor de ferro é um

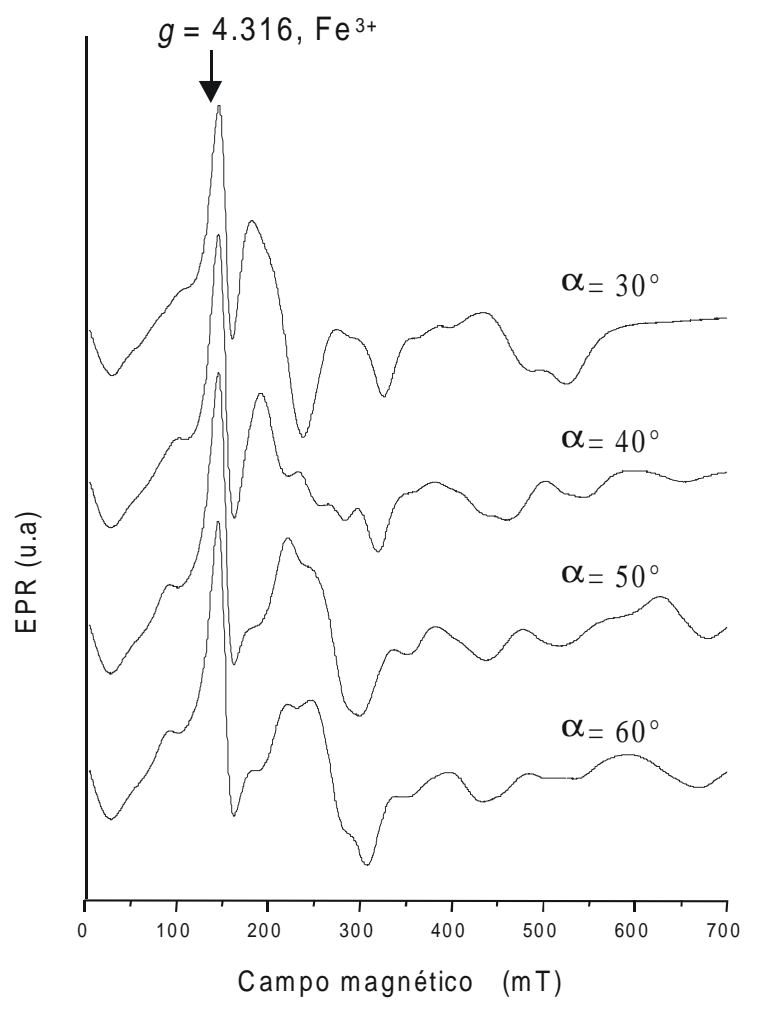

Figura 3- Espectro de ressonância paramagnética eletrônica convencional de uma schorlita, medido a $30 \mathrm{~K}$ e $1 \mathrm{~mW}$ em diferentes orientações.

Tabela 3 - Parâmetros hiperfinos derivados dos espectros Mössbauer das Fe-turmalinas após tratamento. Desvio isomérico, d (mm/ $\mathrm{s}$, relativo ao a-Fe), desdobramento quadrupolar, $\mathrm{D} E_{Q}(\mathrm{~mm} / \mathrm{s})$, largura a meia altura, $\mathrm{G}(\mathrm{mm} / \mathrm{s})$ e área relativa, $R A(\%), T$ tratamento térmico.

\begin{tabular}{ccccccc}
\hline \multirow{2}{*}{ AM OSTRA } & $\chi 2$ & $\Delta E_{\mathrm{Q}}$ & $\boldsymbol{\delta}$ & $\boldsymbol{\Gamma}$ & $\mathrm{RA} \%$ & $\mathrm{~A}$ tribuição \\
\hline \multirow{2}{*}{ CAAC(T) } & 1762 & 2,443 & 0,964 & 0,33 & 18,56 & $\mathrm{Fe}^{2+} \mathrm{Y} 1$ \\
& & 2,393 & 1,097 & 0,33 & 63,10 & $\mathrm{Fe}^{2+} \mathrm{Y} 2$ \\
\cline { 2 - 6 } C AVC(T) & 0,548 & 0,466 & 0,44 & 18,34 & $\mathrm{Fe}^{3+}$ \\
& 1672 & 2,527 & 1,066 & 0,31 & 30,87 & $\mathrm{Fe}^{2+} \mathrm{Y} 1$ \\
& & 2,351 & 1,098 & 0,30 & 44,72 & $\mathrm{Fe}^{2+} \mathrm{Y} 2$ \\
& 1,705 & 1,087 & 0,56 & 24,41 & $\mathrm{Fe}^{2+} \mathrm{Y} 3$ \\
\hline
\end{tabular}


pouco maior na azul, assim como o grau de desordem. Corroborando essa observação, o grau de desordem na amostra verde, após aquecimento, também aumenta com a existência do terceiro sítio Y3 e o matiz da cor resultante é muito próximo do produzido a temperaturas inferiores na amostra azul (Castañeda 2002).

Os espectros Mössbauer das amostras CAAC e CAAV após tratamento térmico em ambiente redutor não puderam ser ajustados devido à presença de uma fase adicional rica em ferro produzida durante esse processo (Fig. 5). Em ambas amostras essa fase é magnética à temperatura ambiente. Nenhum aspecto espectroscópico da presença de maghemita, hematita e magnetita, comuns em espectroscopia Mössbauer sob baixa temperatura, foi identificado para possível correlação com a fase adicional. Foram feitas, também, tentativas de identificação por meio de difração de raios-X, porém sem sucesso. De fato, a presença de uma segunda fase é constatada mas, até o presente momento, pode-se apenas suspeitar que se trata de inclusões microscópicas ricas em ferro e magnéticas. Além disso, pode tratar-se de fase desconhecida, cristalina ou não.

Apesar do comportamento do ferro não ter sido caracterizado completamente, é constatada a presença dominante de $\mathrm{Fe}^{2+}$ pelo dupleto assimétrico (nenhum $\mathrm{Fe}^{3+}$ ). Além disso, era de se esperar um comportamento análogo ao das amostras naturais, uma vez que as cores naturais não foram afetadas significativamente. Entretanto, pequenas perdas de ferro podem ser consideradas devido à geração da fase adicional.

CONCLUSÕES Nos espectros Mössbauer, o maior número de sítios Y não equivalentes e a extensa faixa da distribuição dos valores do desdobramento quadrupolar encontrada indicam grau de desordem da estrutura que pode favorecer o condicionamento de determinados centros cromóforos. Esses aspectos estruturais estão diretamente relacionados com a compensação de diferentes cargas e o efeito geométrico das substituições, considerando a heterogeneidade local. De acordo com essa premissa, os diferentes comportamentos dos componentes dominantes dos dupletos de ferro observados nos dois conjuntos de cores, azul e verde, podem ser indícios de diferentes construções de centros cromóforos relativos ao $\mathrm{Fe}^{2+}$ e mostram que as turmalinas azuis são mais desordenadas que as verdes. Os resultados obtidos nas
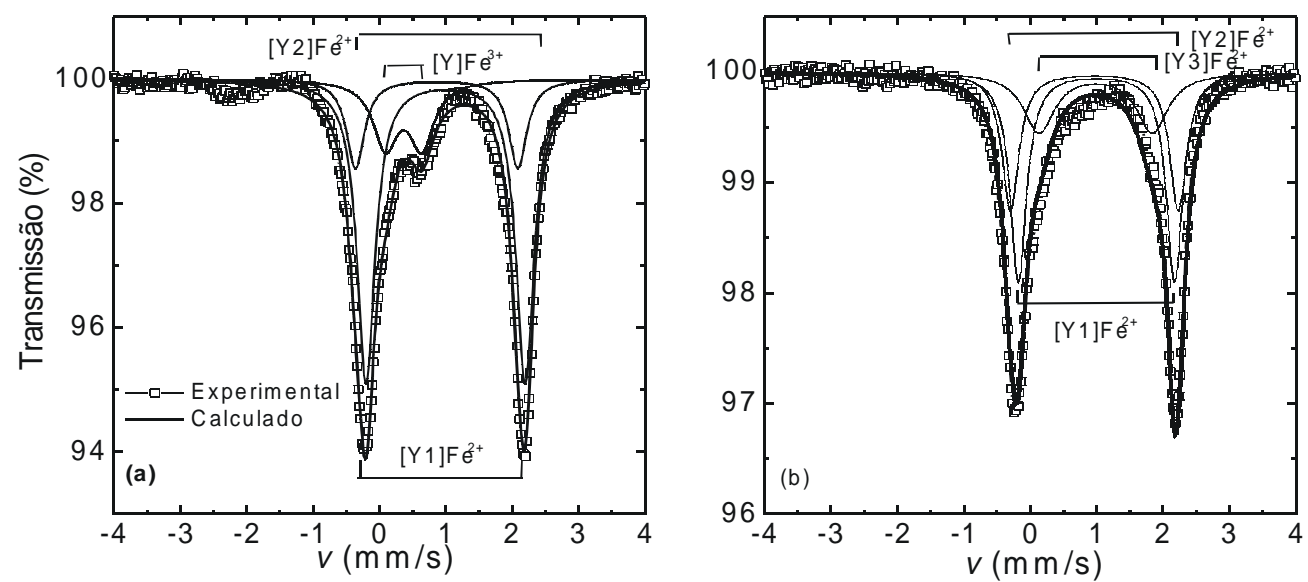

Figura 4 - Espectros Mössbauer sob temperatura ambiente, após tratamento térmico com aquecimento a $700^{\circ} \mathrm{C}$, das amostras azul claro CAAC (a) e verde claro CAVC (b),
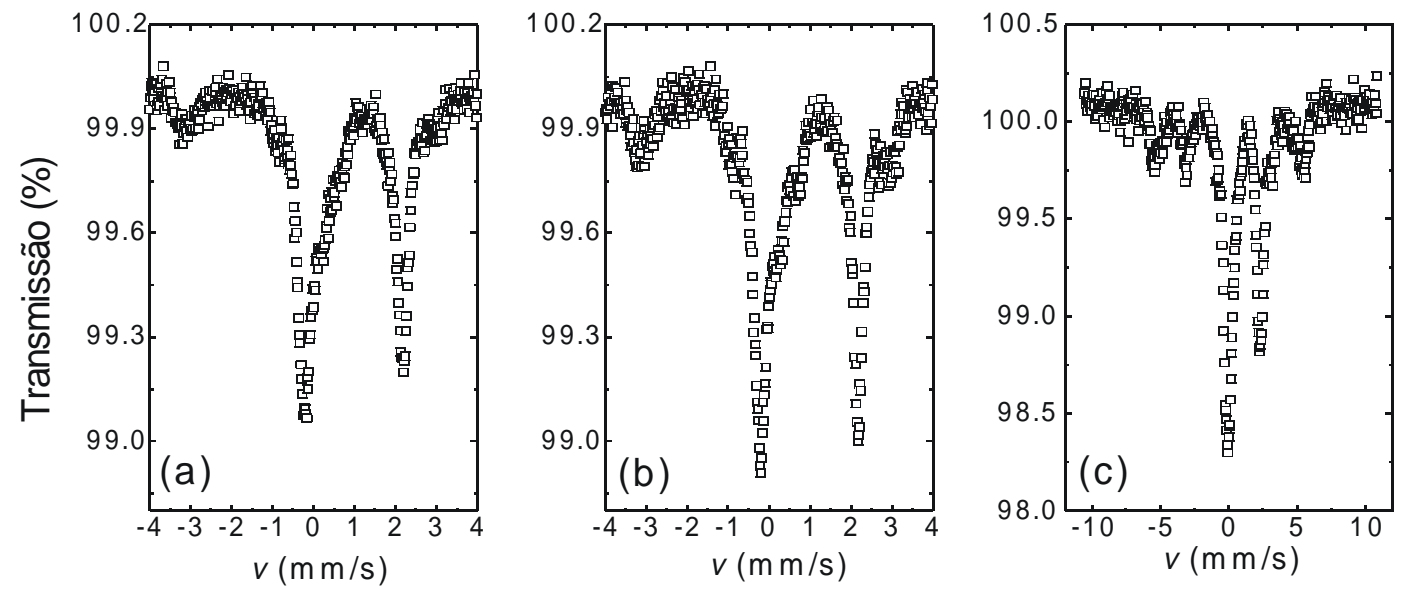

Figura 5 - Espectros Mössbauer sob temperatura ambiente, após aquecimento em ambiente redutor, das amostras CAAC (a) e CAVC (b) e espectro Mössbauer a $80 \mathrm{k}$ da amostra CAAC (c). 
amostras estudadas são semelhantes aos dados de amostras de turmalinas verdes e azuis de outras ocorrências e proveniências, cujos espectros Mössbauer também mostram o posicionamento do $\mathrm{Fe}^{2+} \mathrm{em}$ sítios Y não equivalentes (Oliveira et al. 2002).
Agradecimentos Este trabalho é parte da tese do primeiro autor que agradece ao CNPq pela concessão de uma bolsa de doutorado e a Universidade de Gent, Bélgica, pelo suporte laboratorial. Aos revisores da RBG pelas sugestões ao manuscrito.

\section{Referências}

Barton R. 1969. Refinement of the crystal structure of buergerite and the absolute orientation of tourmalines. Acta Cryst.., B(25):1524-1533.

Bloodaxe E.S., Hughes J.M., Dyar M.D., Grew E.S., Guidotti C.V. 1999. Linking structure and chemistry in the Schorl-Dravite series. Am. Mineral., 84:922-928.

Bonazzi P. \& Bindi L. 1999. Structural adjustments induced by heat treatment in ilvaite. Am. Mineral., 84:1604:1612.

Burns R.G. 1972. Mixed valiances and site occupancies of iron in silicate minerals from Mössbauer spectroscopy. Can. J. Spectroscopy, 17:5159

Burns R.G. 1982. The blackness of schorl: $\mathrm{Fe}(2+) \mathrm{Fe}(3+)$ electron delocalization in tourmaline. Trans. Am. Geophys. Union, 64:1142.

Burns P.C., MacDonald D.J., Hawthorne F. 1994. The crystal chemistry of manganese-bearing elbaite .Can. Mineral., 32:31-41.

Castañeda C. 2002. Caracterização mineralógica de amostras naturais e tratadas de turmalinas e morganitas do Distrito Pegmatítico de Araçuaí, Minas Gerais. Instituto de Geociências, Universidade de Brasília, Brasília, Tese de Doutoramento, 187p.

Castañeda C., César-Mendes J., Pedrosa-Soares A.C. 2001. Turmalinas. In: C. Castañeda, J.E. Addad, A. Liccardo (eds.) Gemas de Minas Gerais. Brasil, SBG-MG, pp.: 152-179.

Da Costa G.M., Castañeda C., Eeckhout S.G., De Grave E., Gomes N.S. 1998. The temperature dependence of the hyperfine parameters of tourmalines. Hyperfine Interations, 3C:344-347.

De Grave, E. \& Van Alboom, A. 1991. Evaluation of ferrous and ferric Mössbauer fraction. Phys. Chem. Minerals, 18:337-342.

Dyar, M.D., Taylor, M.E., Lutz, T.M., Francis, C.A. Guidotti, C.V., Wise, M. 1998. Chemical characterization of tourmaline: Mössbauer study of Fe valence and site occupancy. Am. Mineral., 83:848-864.

Donnay, G. \& Barton, R. 1972. Refinement of the crystal structure of elbaite and the mechanism of tourmaline solid solution. Tschermaks Min. Petr. Mitt, 18:273-286.

Epprecht W. 1953. Die Gitterkonstanten der Tourmaline. Schweiz. Min. Petr. Mitt., 33:481-505.

Faye G.H., Manning P.G., Gosselin J.R. 1974. The optical absorption spectra of tourmaline: importance of charge-transfer processes. Can. Mineral., 12:370-380.

Ferrow E.A., Annersten H., Gunawardane R.P. 1988. Mössbauer effect study on the mixed valence state of iron in tourmaline. Mineral. Mag., 52:221-228.

Foit Jr. F.F. \& Rosenberg P.E. 1977. Coupled substitutions in the tourmaline group. Contrib. Mineral. Petrol., 62:109-127.

Foit Jr. F.F. \& Rosenberg P.E. 1979. The structure of vanadium-bearing tourmaline and its implications regarding tourmaline solid solutions. Am. Mineral., 64:788-798.

Fortier S. \& Donnay G. 1975. Schorl refinement showing composition dependence of the tourmaline structure. Can. Mineral., 13:173-177.

Fuchs Y., Lagache M., Linares J. 1998. Fe-tourmaline synthesis under different $T$ and $\mathrm{fO}_{2}$ conditions. Amer. Mineral., 83:525-534.

Grice J.D. \& Ercit T.S. 1993. Ordering of Fe and Mg in the tourmaline crystal structure: the correct formula. N. Jb. Miner. Abh., 165:245-266.

Hawthorne, F.C. 1996. Structural mechanisms for light-element variations in tourmaline. Can. Mineral., 34:123-132.

Hawthorne F.C. \& Henry D.J. 1999. Classification of the minerals of the tourmaline group. Europ. J. Mineral., 11:201-215.

Hawthorne F.C., MacDonald D.J., Burns P.C. 1993. Al/Mg disorder in crystal structure of dravite. Am. Mineral., 78:265-270

Ishida K. 1998. Cation disordering in heat-treated anthophyllites trough oxidation and dehydrogenation. Phys. Chem. Minerals, 25:160-167.

MacDonald, D.J. \& Hawthorne, F.C. 1995. The crystal chemistry of Si «Al substitution in tourmaline. Can. Mineral., 33:849-858.

Mattson S.M. \& Rossman G.R. 1987a. Identifying characteristic of charge transfer transitions in minerals. Phys. Chem. Minerals, 14:94-99.

Mattson, S.M. \& Rossman, G.R. 1987b. $\mathrm{Fe}^{2+}-\mathrm{Fe}^{3+}$ interactions in tourmalines. Phys. Chem. Minerals, 14:163-171.

Oliveira E.F., Castañeda C., Eeckhout S.G., Gilmar M.M., Kwitko R.R., De Grave E., Botelho N.F. 2002. Infrared and Mössbauer study of brazilian tourmalines from different geological environments. Am. Mineral., 87:1154-1163.

Pieczka A. 1999. Statistical interpretation of structural parameters of tourmalines: the ordering of ions in the octahedral sites. Euro. J. Mineral., 11:243-251.

Pieczka A . 2000. Modelling of some structural parameters of tourmalines on the basis of their chemical composition. I. Ordered structure model. European Journal of Mineralogy, 12:589-596.

Pieczka A., Kraczka J., Zabinski W. 1997. Mössbauer spectra of $\mathrm{Fe}^{3+}$ poor schorls: reinterpretation of the spectra on a basis of an ordered structure model. In: International Symposium on Tourmaline Tourmaline 1997. Nové Mesto na Morave, Czech Republic, Abstract, 74-75.

Povondra P. 1981. The crystal chemistry of tourmalines of the schorldravite series. Acta Universitatis Carolinae - Geologica, 3:223-264.

Rancourt D.G. 1994. Mössbauer spectroscopy of minerals. I. Inadequacy of Lorentzian-line doublets in fitting spectra arising from quadrupole splitting distributions. Phys. Chem. Minerals, 21:244-249.

Ruby, S.L. 1973. Why misfit when you already have c2? In: I.J. Gruverman \& C.W. Seidel (Eds.) Mössbauer Effect Methodology, 8, Plenum Press, New York, pp.: 263-276.

Vandenberghe R.E., De Grave E., Bakker P.M.A. 1994. On the metodology of the analysis of Mössbauer spectra. Hyperfine Interactions, 83:29-49.

Manuscrito A-1359

Recebido em 01 de maio de 2002

Revisão dos autores em 20 de fevereiro de 2003 Revisão aceita em 25 de fevereiro de 2003 